malignidade. Conclusão: a ultra-sonografia é um método diagnóstico que pode ajudar na diferenciação de tumores sólidos benignos e malignos. A presença de contornos irregulares, ecos internos heterogêneos, ligamentos de Cooper espessados e aumento do diâme- tro ântero-posterior podem indicar maior probabilidade de malignidade nos nódulos sólidos da mama.

PALAVRAS-CHAVE: Mama; Câncer de mama; Nódulo de mama

Resumo de Tese

\title{
Influência nas taxas de implantação, da seleção de pré-embriões para transferência uterina, a partir do escore dos pró-nucleados associado ao obtido no terceiro dia de cultura
}

\author{
Influence of embryoselection based on the pronuclear morphology associated with the \\ morphology obtained after 72 hours of culture in the implantation rates
}

Nilka Fernandes Donadio

Orientador: Prof. Dr. Nilson Donadio

Tese de Mestrado apresentada ao Departamento de Obstetrícia e Ginecologia da Irmandade da Santa Casa de Misericórdia de São Paulo, em 5 de Janeiro de 2005.

Com o objetivo de reduzir as gestações múltiplas e a conseqüente mortalidade neonatal, têm-se estabelecido, em fertilização "in vitro", novos critérios de seleção que permitem a transferência de menor número de pré-embriões (PEs) para o útero, sem redução nas taxas de gestação. Assim, 300 casais foram submetidos à injeção intracitoplasmática de espermatozóides, para se avaliar a influência, nas taxas de implantação, da seleção de pré-embriões a partir do escore morfológico obtido na fase de pró-nucleado somado ao do terceiro dia de cultivo (D3). As mulheres foram distribuídas, 75 delas no subgrupo denominado A1, para qual foram transferidos até quatro PEs selecionados exclusivamente pela morfologia no D3; outras 75 , no subgrupo A2, com transferência de até dois PEs selecionados da mesma forma. Para o subgrupo B1, também com 75 mulheres, foram transferidos até 4 PEs selecionados pelo somatório do escore morfológico do pró-nucleado com o obtido em D3. O mesmo critério foi utilizado para o subgrupo B2, limitando-se a trans- ferência em até dois PEs. Na morfologia do pró-nucleado foi avaliada a localização, tamanho e justaposição dos pró-núcleos, distribuição e número dos corpúsculos precursores dos nucléolos e, por fim, a presença de halo citoplasmático. Encontramos taxas de implantação nos subgrupos A1, A2, e B1 de 17, 17 e 19\% respectivamente, as quais não apresentaram diferenças estatísticas entre sí. Em contrapartida, no subgrupo B2, a taxa de implantação foi significativamente superior, chegando a 27\%. Quanto às taxas de gestação clínica, não houve diferenças estatísticas entre os quatro grupos $(p=0,429)$. Concluiu-se que a seleção de pré-embriões baseada na morfologia do pró-nucleado associada a obtida após sua evolução até o terceiro dia de cultivo promove um aumento das taxas de implantação, devendo ser adotada como rotina nos centros de Reprodução Assistida.

PALAVRAS-CHAVE: Infertilidade; Fertilização in vitro; Zigoto; Implantação

Resumo de Tese

\section{As intercorrências clinicas, obstétricas e os resultados perinatais em grávidas portadoras de} transplante renal

\section{The clinical and obstetric reports and perinatal outcomes in pregnant womem with renal transplantation}

Autor: Leandro Gustavo de Oliveira

Orientador: Prof. Dr. Nelson Sass

Co-orientador: Prof. Dr. Luiz Camano

Tese apresentada à Universidade Federal de São Paulo - Escola Paulista de Medicina, para obtenção do Título de Mestre em Ciências, em 8 de novembro de 2004. 
Objetivo: avaliar as inter-relações recíprocas entre o transplante renal e a gravidez por meio da análise das intercorrências clínicas e obstétricas, bem como da investigação do desfecho perinatal. Métodos: foi analisada uma série retrospectiva de 39 casos de gestação em 37 portadoras de transplante renal no período de janeiro de 1997 a dezembro de 2003. As variáveis estudadas foram relativas ao transplante, às intercorrências clínicas e obstétricas e aos resultados perinatais. Um grupo controle composto por 66 grávidas sem patologias prévias, que freqüentavam pré-natal e deram à luz em 2002 e 2003 foi estabelecido para possíveis avaliações de significância estatística. Foram utilizados os testes de qui quadrado $\left(\chi^{2}\right)$ e exato de Fisher. Resultados: a média de idade das pacientes no momento da concepção foi de 27 anos. O doador vivo foi o mais freqüente. A ocorrência de disfunção do enxerto foi de $47,4 \%$, tendo sido a pré-eclampsia a sua principal causa. Perda do transplante ocorreu em 10,2\% dos casos. Entre os imunossupressores, a ciclosporina foi o mais utilizado. Hipertensão arterial crônica incidiu em $82 \%$ dos casos, anemia em $77 \%$ e infecção do trato urinário em $38,5 \%$. Quanto à via de parto, a cesariana foi realizada em $53,8 \%$, tendo como principal indicação a insuficiência placentária. A freqüência de préeclampsia foi de $28,2 \%$. Quanto às variáveis perinatais a prematuridade foi constatada em $46,1 \%$ dos casos, apresentando relação significativa com níveis de creatinina iguais ou superiores a $1,5 \mathrm{mg} / \mathrm{dL}$ no primeiro trimestre da gestação. Não houve relação com o uso de ciclosporina. Outra intercorrência observada foi restrição de crescimento fetal, verificada em $41,0 \%$ das gestações, não apresentando relação com os níveis iniciais de creatinina ou uso de ciclosporina. Conclusões: $\mathrm{O}$ grupo de estudo foi constituído por pacientes jovens. Disfunção do enxerto foi freqüente, havendo também casos de perda do transplante. Hipertensão arterial crônica, anemia e infecção do trato urinário foram as intercorrências clínicas mais freqüentes. Quanto à via de parto a cesariana teve incidência elevada. Pré-eclampsia ocorreu de forma significativamente maior que no grupo controle. Prematuridade e restrição de crescimento fetal foram as principais complicações perinatais.

PALAVRAS-CHAVE: Transplante renal; Imunossupressão; Pré-eclâmpsia

\section{Estudo da prevalência dos fatores de risco do câncer de mama, numa população de funcionárias da Maternidade Escola Assis Chateaubriand da Universidade Federal do Ceará}

Prevalence of risk factors for breast cancer study in a group of the female staff from the
school maternity hospital Assis Chateaubriand (Federal University of Ceará, Brazil)

Autor: Ércio Fereira Gomes

Orientador: Prof. Dr. Luiz Gonzaga Porto Pinheiro

Dissertação apresentada à Coordenação do Curso de Pós-Graduação em Tocoginecologia, da Universidade Federal do Ceará, como requisito parcial para obtenção do título de Mestre em Ginecologia e Obstetrícia, em 22 de dezembro de 2004

Para determinar a prevalência de alguns fatores de risco do câncer de mama, quantificar os fatores de risco de maior prevalência e delimitar grupos de maior risco, analisou-se uma população de 425 funcionárias da Maternidade Escola Assis Chateaubriand da Universidade Federal do Ceará, de vários níveis sociais e padrões raciais, mediante a aplicação de um formulário com o levantamento de 30 variáveis e realizou-se a mensuração do peso, altura, circunferência da cintura e do quadril. Calculou-se com estes dados a relação cintura/quadril, o índice de massa corpórea e o índice de Gail de todas as entrevistadas. Dos fatores de risco levantados, encontrou-se em 222 funcionárias $(52,2 \%)$ o índice de massa corpórea acima de 25 , a relação cintura/quadril maior do que 0,8 em 391 (92,1\%) e o sedentarismo em $314(73,1 \%)$ das entrevistadas. O grupo de maior risco encontrado e que merece seguimento diferenciado é formado pelos seguintes subgrupos: as $222(52,2 \%)$ funcionárias com o índice de massa corpórea maior ou igual a 25 , as $34(11,3 \%)$ que tiveram o primeiro filho após os trinta anos, as 34 $(11,3 \%)$ que fazem ou fizeram terapia de reposição hormonal, as $2(0,5 \%)$ portadoras de câncer de mama e as $15(20,8 \%)$ com parentes de primeiro grau acometidos de câncer de mama.

PALAVRAS-CHAVE: Neoplasias mamárias - fatores de risco. Neoplasias mamárias - grupos de risco. Câncer: rastreamento. 